\title{
Predictive value of ultrasound diagnosis of aberrant right subclavian artery with non-recurrent laryngeal nerve
}

Rong-Xi Liang ${ }^{1}$, Bo Wang ${ }^{2}$, Wen-Xin Zhao ${ }^{2}$ En-Sheng Xue ${ }^{1}$, Qin Ye ${ }^{1}$, Zhi-Yong Chen ${ }^{3}$, Zhi-Kui Chen ${ }^{1}$, Xue-Ying Lin ${ }^{1}$, Zhen-Hu Lin ${ }^{1}$, You-Jia Lin ${ }^{1}$

${ }^{1}$ Department of Ultrasound, Union Hospital of Fujian Medical University, Fuzhou 350001, Fujian Province, China

${ }^{2}$ Department of Vessels and Thyroid Surgery, Union Hospital of Fujian Medical

University, Fuzhou 350001, Fujian Province, China

${ }^{3}$ Department of Radiology, Union Hospital of Fujian Medical University,

Fuzhou 350001, Fujian Province, China

Submitted: 6 September 2019

Accepted: 28 November 2019

Arch Med Sci

DOI: https://doi.org/10.5114/aoms.2020.98971

Copyright (c) 2020 Termedia \& Banach

\section{Abstract}

Introduction: This study aims to evaluate the predictive value of color Doppler ultrasound for the diagnosis of aberrant right subclavian artery (ARSA) with a co-occurring non-recurrent right laryngeal nerve (NRLN).

Material and methods: In the present study, 58 patients with ARSA (ARSA group) and 1,280 patients without ARSA (controls) were diagnosed by ultrasonography. In addition, 32 patients with ARSA (ARSA operation group) and controls underwent thyroidectomy with surgical exploration with or without NRLN. Then, the incidence of NRLN was analyzed. The right common carotid artery (RCCA) and right subclavian artery (RSA) trends were observed by ultrasound, and classified into two types: RCCA and RSA originating from the innominate artery (IA) (type I), and IA could not be detected (type II).

Results: A total of 32 cases of NRLN were found in the ARSA operation group, but no case was found in controls, and the difference was statistically significant $(p=0.0006)$. The difference in the constituent ratio of type I and type II was statistically significant between the ARSA group and controls $(p=0.0002)$. That is, the IA could not be detected in the ARSA group, which was accompanied by the RCCA that originated from the aortic arch, while the IA was detected in most patients in the control group at the level of the sternoclavicular joints.

Conclusions: Aberrant right subclavian artery can be rapidly detected by ultrasonography. Aberrant right subclavian artery occurs when the RCCA originates from the aortic arch during detection. Patients with ARSA sometimes have co-occurring NRLN. Hence, vigilance in protecting the NRLN is needed during an operation.

Key words: ultrasonography, aberrant right subclavian artery, ARSA, nonrecurrent right laryngeal nerve, NRLN.

\section{Introduction}

The aberrant subclavian artery (ASA) is a rare anatomical variant, which can be divided into two types: aberrant right subclavian artery (ARSA), and aberrant left subclavian artery (ALSA). An ARSA is more

\section{Corresponding author:} En-Sheng Xue Union Hospital of Fujian

Medical University No.29 of Xinquan Road Gulou District

Fuzhou 350001 Fujian Province, China E-mail:

enshengxue1071@163.com 
common than ALSA, with a reported incidence of $0.19-2.52 \%$ of the healthy population [1-3].

The non-recurrent laryngeal nerve is the nerve that does not loop around the right subclavian artery (RSA) on the right side or go around the aortic arch on the left side, but directly enters into the laryngeal after branching off from the vagus nerve [3-6]. As a result, the anatomic variant of the non-recurrent laryngeal nerve may easily cause recurrent laryngeal nerve injury during an operation, especially in the absence of intraoperative monitoring and locating on the recurrent laryngeal nerve, leading to a rate of recurrent laryngeal nerve injury as high as $16.8 \%$ [6-8]. Furthermore, the patient would have a worse quality of life, such as postoperative glottal obstruction, and coughing on intake of food and water, as a result of the recurrent laryngeal nerve injury. Therefore, there is important clinical significance in protecting the non-recurrent laryngeal nerve during thyroid or parathyroid surgery.

The recurrent laryngeal nerve is closely associated with the subclavian artery, common carotid artery (CCA) and aortic arch during embryonic development. Thus, the developmental anomaly of the subclavian artery and CCA, such as ASA, suggests a great possibility of an ipsilateral non-recurrent laryngeal nerve. If an aberrant subclavian artery is identified by a preoperative ultrasound examination, it predicts the high possibility of an ipsilateral non-recurrent laryngeal nerve [5, 9-14].

Therefore, we conducted this study to evaluate the predictive value of color Doppler ultrasound (CDU) for the diagnosis of ARSA with a co-occurring NRLN.

\section{Material and methods}

\section{Patients}

This retrospective analysis included $16,180 \mathrm{pa}$ tients who underwent CDU examinations of the thyroid, cervical lymph node, bilateral common carotid arteries, bilateral subclavian arteries, ascending aorta and aortic arch at our department in the period from January 2010 to May 2018 (6,862 males and 9,318 females). A total of 58 patients were diagnosed with ARSA based on the ultrasound results, and 32 of these patients underwent thyroidectomy as a result of thyroid nodules (ARSA operation group). Furthermore, 1,280 patients without ARSA underwent thyroidectomy for thyroid nodules. Among them, 374 cases were diagnosed as benign thyroid nodules with larger size, and 906 cases were diagnosed as malignant thyroid nodules, by ultrasonography respectively. No right-sided aortic arch with ALSA was found, which was further confirmed by computed tomography (CT) (11697 cases) or magnetic reso- nance angiography (MRA) (4,483 cases). The present study was approved by the Ethics Committee of our hospital, and all patients provided signed informed consent.

Meanwhile, surgical exploration was performed for patients with or without NRLN during the thyroidectomy. All procedures were reviewed by the Institutional Ethics Committee and approved by the patients.

\section{Instruments and methods}

All patients were evaluated by experienced observers using a high-frequency probe $(7.5-14 \mathrm{MHz})$, transabdominal probe $(3.5-5.0 \mathrm{MHz})$, intracavitary probe $(5.0-7.5 \mathrm{MHz})$, or cardiac probe $(2-5 \mathrm{MHz})$ through an ultrasound instrument (Toshiba 500, Acuson Sequia 512, Supersonic Aixplorer), as appropriate.

Then, the echogenicity of the thyroid and cervical lymph node metastasis or nodes without metastasis was evaluated. Meanwhile, the arterial trends of the bilateral common carotid arteries, bilateral subclavian arteries, ascending aorta, and aortic arch were observed with the probes, as appropriate. The diameter of the right common carotid artery (RCCA) was measured by CDU with a high frequency probe at the level of the sternoclavicular joints for all groups. Then, the RCCA and RSA trends were observed by dynamic ultrasound. According to the arterial trend, and as detected by CDU using a high frequency probe, these patients were divided into two groups: the RCCA and RSA originated from the innominate artery (IA) (type I); the IA could not be detected and the RCCA originated directly from the aortic arch or common stem with the left common carotid artery (LCCA) originating from the aortic arch (type II). The type I and type II ratio and RCCA diameter were compared between the ARSA group and control group.

\section{Statistical analysis}

The software program SPSS 13.0 was used to perform the statistical analysis. Continuous variables were expressed as mean \pm standard deviation (SD). Discontinuous variables were expressed as percentage (\%). For two-sample comparisons, groups were compared by $t$-test when each data set conformed to the normal distribution, while non-normally distributed continuous data were compared using non-parametric tests. $P<0.05$ was considered statistically significant.

\section{Results}

\section{General information}

A total of 58 patients were diagnosed with ARSA based on the ultrasound results, and 32 of 

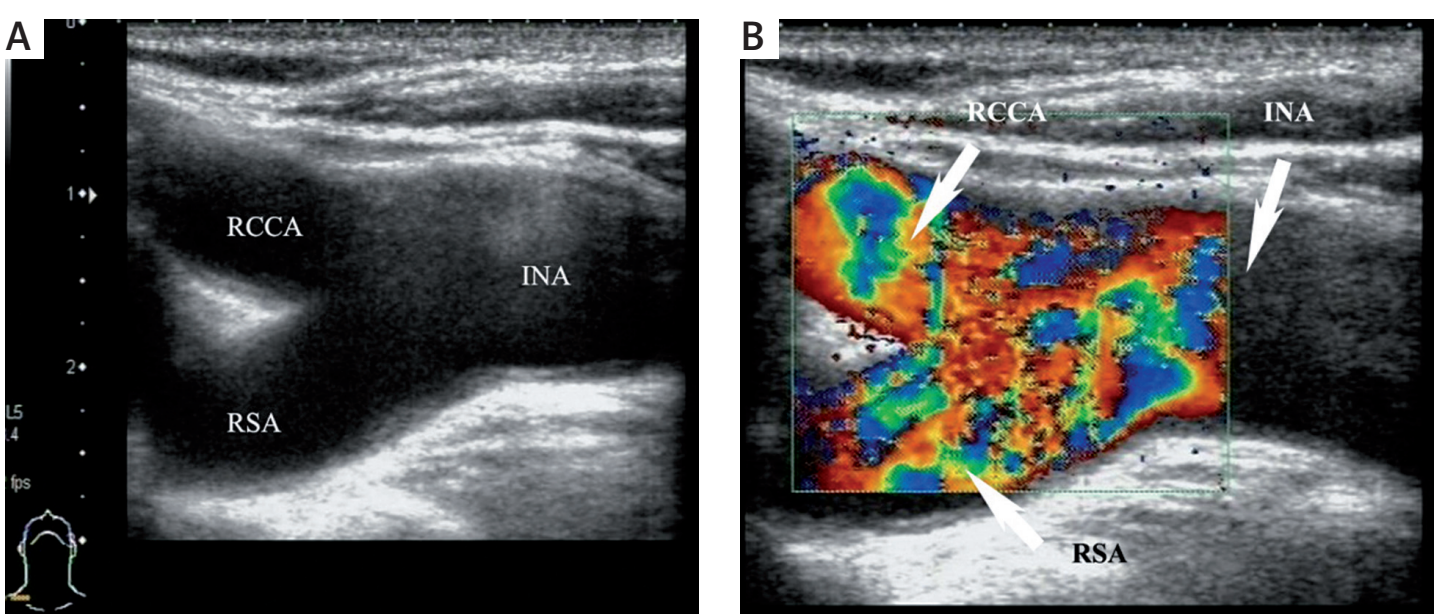

Figure 1. Control group. Scanning with high-frequency probe at the level of sternoclavicular joints; the right common carotid artery (RCCA) route and the right subclavian artery (RSA) route gradually get closer to each other in the same plane. INA - innominate artery

these patients underwent thyroidectomy as result of thyroid nodules (ARSA operation group). Furthermore, 1,280 cases without ARSA underwent thyroidectomy for thyroid nodules. No right-sided aortic arch with ALSA was found, which was further confirmed by CT $(11,697$ cases) or MRA $(4,483$ cases).

\section{Ultrasound findings for the control group}

After scanning with a high-frequency probe at the level of sternoclavicular joints, it was found that the RCCA and RSA routes were closer to each other, gradually converging on the same plane (Figure 1). Meanwhile, the RCCA and RSA that originated directly from the IA were found above the level of the sternoclavicular joints in 157 cases, and at the level or below the level of the sternoclavicular joints in 872 cases. However, the RCCA and RSA that originated from the IA could not be detected in 251 cases due to the limitation of the scanning scope of the high-frequency probe. Hence, there were 1,029 cases of type I and 251 cases of type II (Table I).

After scanning with the transabdominal probe, intracavitary probe, or cardiac probe at the level of the sternoclavicular joints, a wider range was detected. This allowed the vessels to be clearly identified in 251 cases, in which the RCCA and RSA that originated from the IA could not be detected with a high-frequency probe. In addition, this clear-
Table I. Comparison of the ratio of category I and category II between the aberrant right subclavian artery group and control group (\%)

\begin{tabular}{|lcc|}
\hline Group & Category I & Category II \\
\hline ARSA group & $0(0 / 58)$ & $100(58 / 58)$ \\
\hline Control group & $80.39(1029 / 1280)$ & $19.61(251 / 1280)$ \\
\hline
\end{tabular}

ly revealed that the RCCA and RSA originated from the IA near the aortic arch, as a result of the shorter IA. The shortest IA identified was $1.3 \mathrm{~cm}$.

\section{Ultrasound findings for the aberrant right subclavian artery group}

The ultrasound examinations revealed the following: (1) 58 cases were diagnosed with ARSA (ARSA group) based on the ultrasound findings, and this was further confirmed by computed tomography angiography (CTA) or MRA, and 32 of these 58 cases underwent thyroidectomy as a result of thyroid nodules (ARSA operation group); (2) 1,280 cases without ARSA underwent thyroidectomy for thyroid nodules (control group); (3) no right-sided aortic arch with ALSA was found, and this was further confirmed by CT (11,697 cases) or MRA (4,483 cases) (Table II and Figure 2).

After scanning with a high-frequency probe at the level of the sternoclavicular joints, it was found that the RCCA and RSA routes were not

Table II. Information on all groups

\begin{tabular}{|lccccc|}
\hline Group & Male & Female & Age - range [years] & Age - mean [years] & Age - SD [years] \\
\hline ARSA group $(n=58)$ & 15 & 43 & $21-66$ & 36.4 & 9.8 \\
\hline ARSA operation group $(n=32)$ & 9 & 23 & $21-55$ & 35.1 & 8.5 \\
\hline Control group $(n=1280)$ & 537 & 743 & $16-73$ & 34.2 & 11.1 \\
\hline
\end{tabular}

ARSA - aberrant right subclavian artery, SD - standard deviation. 


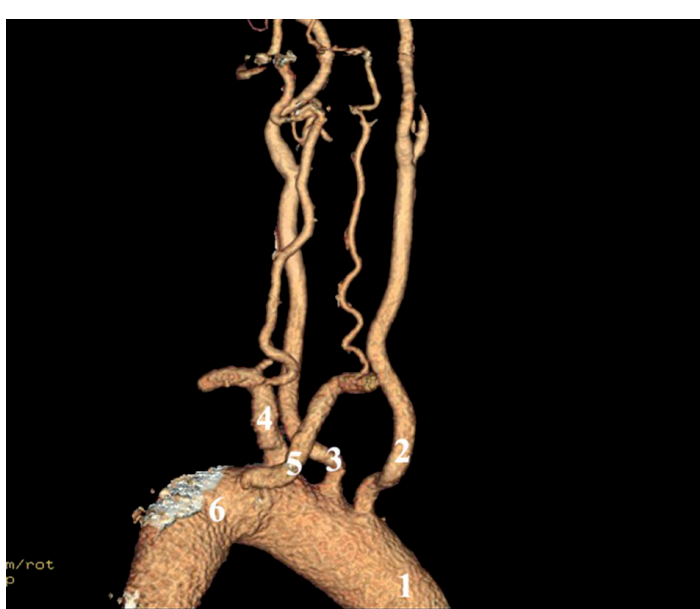

Figure 2. Aberrant right subclavian artery (ARSA) group. Dorsal view: ARSA was confirmed by computed tomography angiography. 1 - ascending aorta, 2 - right common carotid artery, 3 - left common carotid artery, 4 - left subclavian artery, 5 - aberrant ARSA, 6- aortic arch
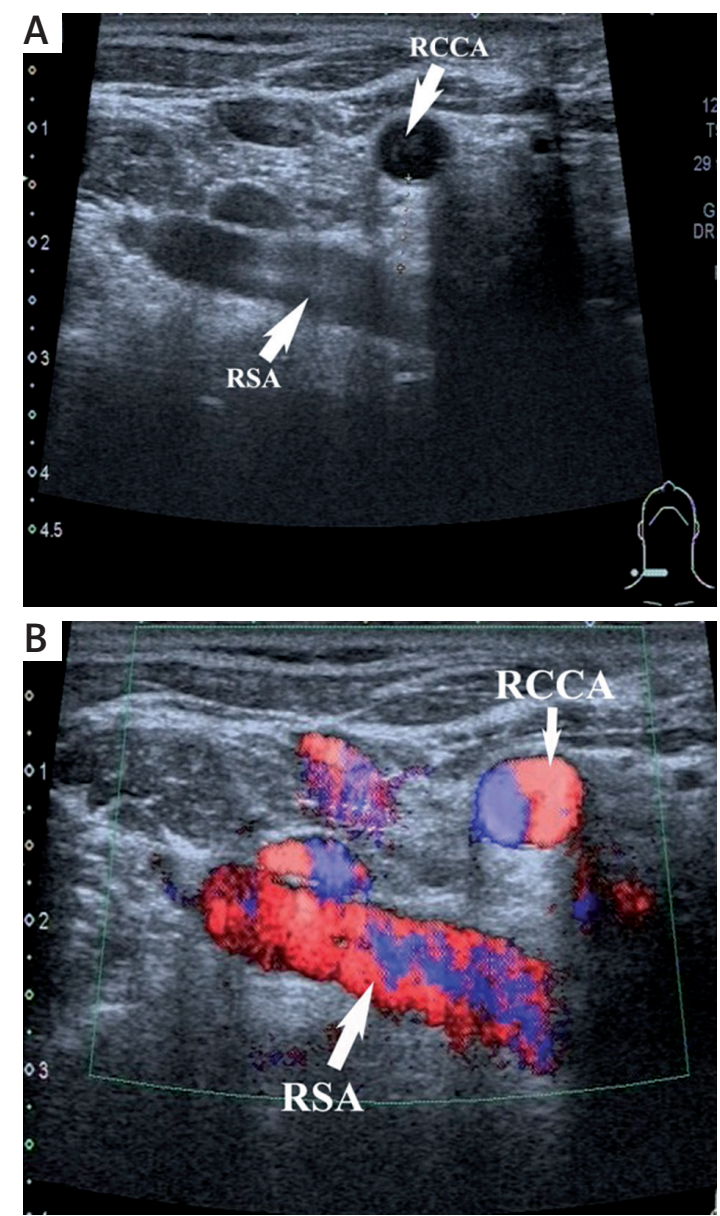

Figure 3. Aberrant right subclavian artery (ARSA) group. Scanning with high-frequency probe at the level of sternoclavicular joints, the right common carotid artery (RCCA) route and the right subclavian artery (RSA) route are not on the same plane throughout; moreover, they separate gradually. They do not originate from the IA within the ultrasonic scanning scope in the same plane, and gradually separated (Figure 3). Furthermore, these did not originate from the IA within the ultrasound scanning scope. That is, there were no cases of type I and 58 cases of type II (Table I). The IA was found to be absent in 46 cases, and the RCCA originated directly from the aortic arch, while for the other 12 cases, this could not be detected due to the far field attenuation of the high-frequency probe due to the short and thick neck of patients.

After scanning with the transabdominal probe, intracavitary probe, or cardiac probe at the level of the sternoclavicular joints, the IA was found to be absent in all cases in the ARSA group (Figure 4). The RCCA and RSA routes gradually separated below the level of the sternoclavicular joints. Furthermore, 53 cases of RCCA that originated directly from the aortic arch were found, while the other five cases had a common stem, with the LCCA originating from the aortic arch.

The type I and type II ratio exhibited a significant difference between the ARSA group and control group ( $p=0.0001, p<0.01$; Table I). After scanning with the ultrasound probe at the level of the sternoclavicular joints, in most cases, the RCCA and RSA that originated from the IA could easily be detected when the two arteries had no course variations. Furthermore, changing the ultrasound probe and enlarging the scanning scope, as appropriate, was necessary when the RCCA and RSA that originated from the IA could not be detected at the level of the sternoclavicular joints. If the RCCA and RSA routes gradually separated below the level of the sternoclavicular joints, a minute examination to the RCCA and RSA is required. Hence, if the RCCA originating directly from aortic arch could be detected, the ARSA has occurred.

\section{Relationship between non-recurrent right laryngeal nerve and aberrant right subclavian artery}

Thirty-two cases of NRLNs were confirmed in the ARSA operation group, while no case of NRLN found in the control group during the operative exploration. The prevalence of NRLN was statistically significantly different between the ARSA operation group and control group $(p=0.0006, p<0.01$; Table III, Figure 5).

Right common carotid artery and aberrant right subclavian artery diameter

The diameter of the RCCA in the ARSA group and control group was $7.66 \pm 0.38$ and $7.56 \pm 0.36$ $\mathrm{mm}$, respectively, and the difference between the two groups was not statistically significant $(p=0.682,>0.05)$. The diameter of the ARSA in the ARSA group and RSA in the control group 

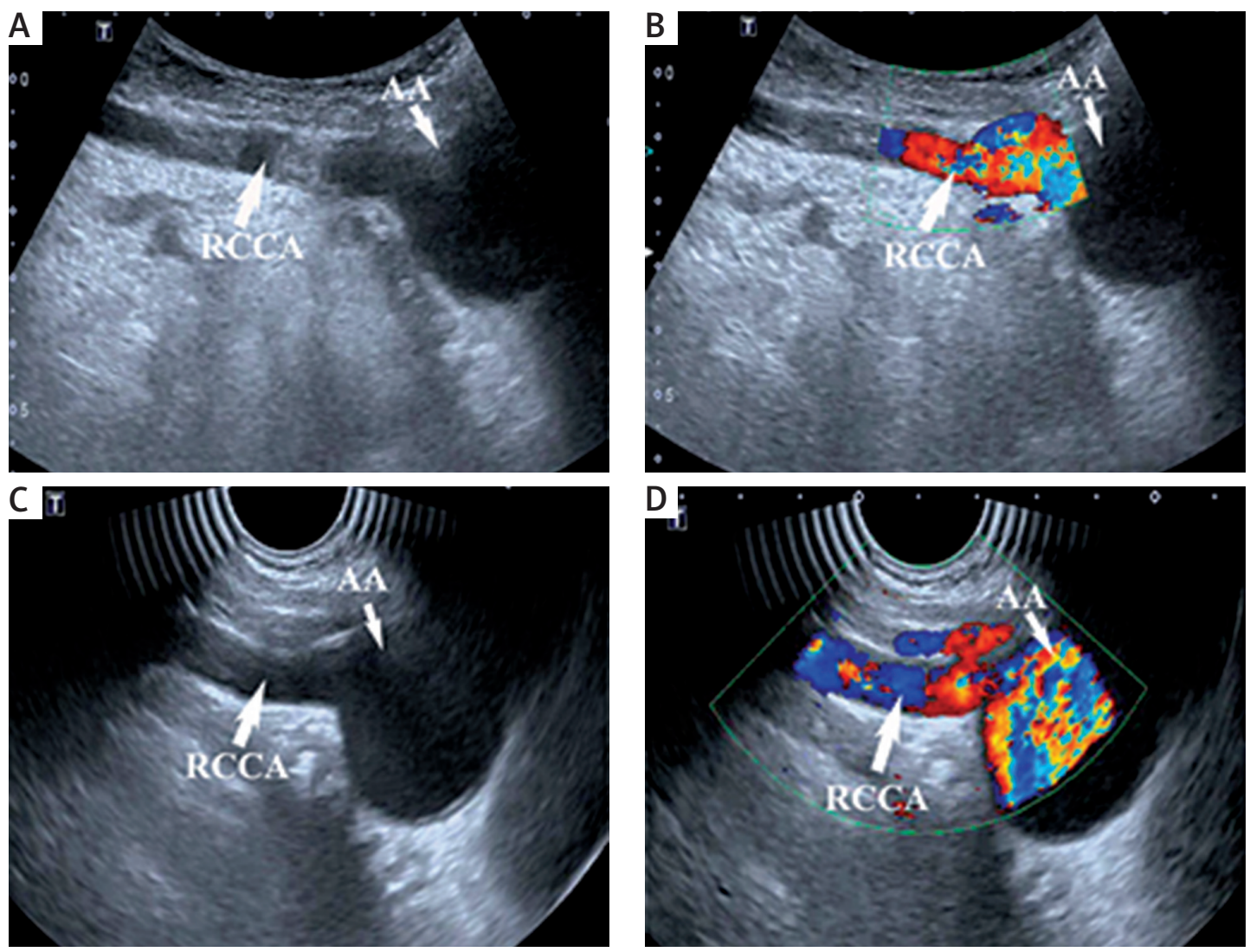

Figure 4. Aberrant right subclavian artery (ARSA) group. A, B - Scanning with transabdominal probe at the level of sternoclavicular joints, right common carotid artery (RCCA) originating directly from aortic arch is found. C, D - Scanning with intracavitary probe at the level of sternoclavicular joints, RCCA originating directly from aortic arch is found and the lumen is more clearly displayed. AA - aortic arch
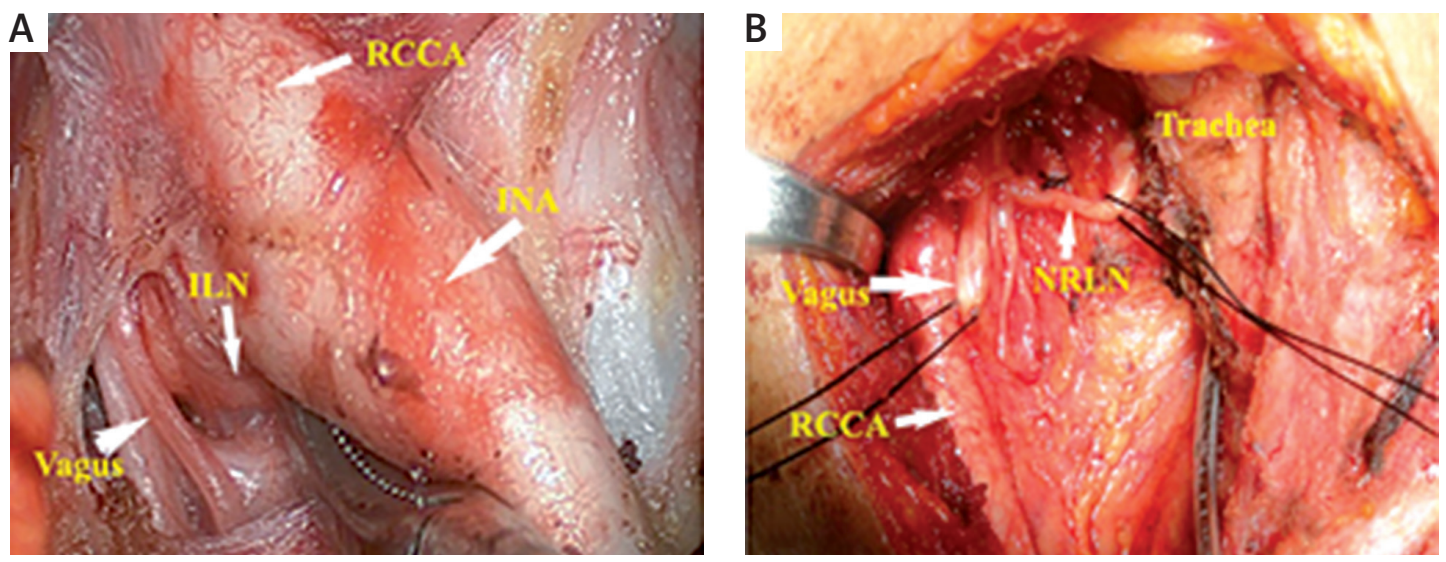

Figure 5. Intraoperative pictures of control group and aberrant right subclavian artery (ARSA) operation group. A - The right recurrent laryngeal nerve (ILN) loops around the right subclavian artery in the control group. B - The nonrecurrent right laryngeal nerve (NRLN) enters into the laryngeal directly after branching off from the vagus nerve in the ARSA operation group. INA - innominate artery, RCCA - right common carotid artery

was $7.79 \pm 0.51$ and $7.68 \pm 0.45 \mathrm{~mm}$, respectively, and the difference between the two groups was not statistically significant $(p=1.961, p>0.05)$.

\section{Discussion}

The outcomes of the present study revealed that 32 cases of NRLN were found in the ARSA op-
Table III. Comparison of prevalence of nonrecurrent right laryngeal nerve (NRLN) between aberrant right subclavian artery (ARSA) operation group and control group

\begin{tabular}{|lcc|}
\hline Group & With NRLN & Without NRLN \\
\hline ARSA operation group & 32 & 0 \\
\hline Control group & 0 & 1280 \\
\hline
\end{tabular}


eration group, but no case was found in the control group. The constituent ratio for type I and type II had a significant difference between the ARSA group and control group. That is, IA could not be detected in the ARSA group, which was accompanied by an RCCA that originated from the aortic arch, while IA was detected in most patients in the control group at the level of the sternoclavicular joints.

Under normal circumstances, the RSA develops from the fourth arterial arcades, the seventh intersegmental artery, and the proximal end of the right aorta dorsalis. For the same reasons, the cranial side of the fourth arterial and right aorta dorsalis abnormally disappeared, but the distal end one, which should degenerate and disappear, remained and subsequently developed into an abnormal subclavian artery with the seventh intersegmental artery, that is, ARSA.

During embryonic development, the development of the recurrent laryngeal nerve is closely correlated with the development of the subclavian artery. The recurrent laryngeal nerve gradually differentiates and develops, accompanied by the differentiation and development of the subclavian artery. Under normal circumstances, after originating from the right vagus, the right recurrent laryngeal nerve goes downward and around the right subclavian artery, goes up along the tracheoesophageal groove, and runs into the larynx at the inferior horn of thyroid cartilage and suspensory ligament of the thyroid gland. For patients with ARSA, as a result of the anomalous artery, the right recurrent laryngeal nerve does not go round the RSA and directly run into the larynx after originating from the right vagus, that is, the NRLN [4-6]. The NRLN is more vulnerable during an operation, as a result of the course variation [7-17]. In the present study, the NRLN was found in all patients with ARSA during the operation, and the prevalence was significantly higher than that in the control group. This shows that more attention in protecting the NRLN should be given to patients with ARSA due to the exceptionally high incidence of NRLN. Through the examination of the bilateral common carotid arteries, bilateral subclavian arteries, ascending aorta and aortic arch, ARSA could be diagnosed preoperatively, reminding the operator to provide more attention to protecting the NRLN during the operation [17-20]. No ALSA was found in this study. Hence, there was no further discussion on that.

In the present study, it was found that ultrasound examination is a convenient method for diagnosing the blood vessel, and allows ARSA to be rapidly, exactly and sensitively diagnosed. Furthermore, there was no significant difference in the diameter of the RCCA between the ARSA group and control group, which shows that the diameter of the RCCA could not be used as an indicator for inferring patients with or without ARSA. However, without the course variation of the RCCA and RSA, $80.39 \%(1029 / 1280)$ of RCCA and RSA that originated directly from the IA were found at the level of the sternoclavicular joints by ultrasound examination with a high-frequency probe. However, the RCCA and RSA routes were not on the same plane, and gradually separated. Hence, extreme vigilance against ARSA is necessary. Hence, a wider range of detection on the course of RCCA and RSA is needed under the level of the sternoclavicular joints with other probes.

For the other methods, due to the limitation of the scanning scope with a high-frequency probe, the subclavian and substernal blood vessel could not be clearly displayed for patients with short and thick necks $[11,12]$. However, by scanning using a transabdominal probe or intracavitary probe at the level of the sternoclavicular joints, a wider range of detection could be achieved due to the lower frequency of the probe, and the subclavian and substernal blood vessels could be clearly displayed. Furthermore, the intracavitary probe, which is smaller, could avoid the blocking of bones, has a slightly higher frequency, and has a wider sector display than that of a transabdominal probe, allowing the subclavian and substernal blood vessels to be more clearly displayed. As a result, regardless of whether the RCCA originated directly from the aortic arch, or whether the short common trunk of the RCCA and LCCA originated from the aortic arch, this could be diagnosed clearly with the intracavitary probe. In the present study, the RSA and RCCA could be clearly displayed by scanning with the intracavitary probe, but these could not be clearly displayed with the high-frequency probe at the level of the sternoclavicular joints, in 12 patients in the ARSA group and 251 patients in the control group. Scanning with the intracavitary probe at the level of the sternoclavicular joints is a simple and efficient method for detecting the subclavian and substernal blood vessels.

Limitations: First, the present study was retrospective in nature, and is not a randomized controlled trial. Second, it was a single-center study, so another trial with multiple centers is still needed. Third, the relationship between ARSA and the non-recurrent laryngeal nerve still needs further research.

In conclusion, aberrant right subclavian artery can be rapidly detected by ultrasonography. Aberrant right subclavian artery occurs when the RCCA originates from the aortic arch during detection. Aberrant right subclavian artery sometimes co-occurs with NRLN. Hence, vigilance in protecting the NRLN is needed during an operation. 


\section{Conflicts of interest}

The authors declare no conflict of interest.

\section{Acknowledgments}

This study was supported by Scientific Research Fund of Fujian Provincial Education Department (No. JA15220).

\section{References}

1. Polednak AP. Prevalence of the aberrant right subclavian artery reported in a published systematic review of cadaveric studies: the impact of an outlier. Clin Anat 2017; 30: 1024-8.

2. Gursoy Erzincan S, Karamustafaoglu Balci B, Tokgoz C, Kalelioglu IH. Incidence of an aberrant right subclavian artery on second trimester sonography in an unselected population. J Ultrasound Med 2017; 36: 1015-9.

3. Natsis K, Didagelos M, Gkiouliava A, Lazaridis N, Vyzas V, Piagkou M. The aberrant right subclavian artery: cadaveric study and literature review. Surg Radiol Anat 2017; 39: 559-65.

4. Henry JF, Audiffret J, Denizot A, Plan M. The nonrecurrent inferior laryngeal nerve: review of 33 cases, including two on the left side. Surgery 1988; 104: 977-84.

5. Kamani D, Potenza AS, Cernea CR, Kamani YV, Randolph GW. The nonrecurrent laryngeal nerve: anatomic and electrophysiologic algorithm for reliable identification. Laryngoscope 2015; 125: 503-8.

6. Masuoka H, Miyauchi A, Higashiyama T, Yabuta T, Kihara M, Miya A. Right-sided aortic arch and aberrant left subclavian artery with or without a left nonrecurrent inferior laryngeal nerve. Head Neck 2016; 38: E2508-11.

7. Defechereux T, Albert V, Alexandre J, Bonnet P, Hamoir E, Meurisse $M$. The inferior non recurrentlaryngeal nerve: a major surgical risk during thyroidectomy. Acta Chir Belg 2000; 100: 62-7.

8. Avisse C, Marcus C, Delattre JF, et al. Right nonrecurrent inferior laryngeal nerve and arteria lusoria: the diagnostic and therapeutic implications of an anatomic anomaly. Review of 17 cases. Surg Radiol Anat 1998; 20: 227-32.

9. Lee JY, Won DY, Oh SH, et al. Three concurrent variations of the aberrant right subclavian artery, the non-recurrent laryngeal nerve and the right thoracic duct. Folia Morphol (Warsz) 2016; 75: 560-4.

10. Gray SW, Skandalakis JE, Akin JT. Embryological considerations of thyroid surgery: developmental anatomy of the thyroid, parathyroids and the recurrent laryngeal nerve. Am Surg 1976; 42: 621-8.

11. Iacobone M, Citton M, Pagura G, Viel G, Nitti D. Increased and safer detection of nonrecurrent inferior laryngeal nerve after preoperative ultrasonography. Laryngoscope 2015; 125: 1743-7.

12. Huang SM, Wu TJ. Neck ultrasound for prediction of right nonrecurrent laryngeal nerve. Head Neck 2010; 32: 844-9.

13. Janardhan D, Patil S, Thomas S, Suresh S. Nonrecurrent laryngeal nerve: a rare but predictable variation. Thyroid Res Pract 2019; 16: 39-41.

14. Varghese BT, Desai KP, Ramachandran A. Pre-operative prediction of a right non-recurrent laryngeal nerve by computed tomography. J Laryngol Otol 2013; 127: 525-7.

15. Wang T, Dionigi G, Zhang D, et al. Diagnosis, anatomy, and electromyography profiles of 73 nonrecurrent laryngeal nerves. Head Neck 2018; 40: 2657-63.
16. Mark NM, Madtes DK. Aberrant right subclavian artery on endobronchial ultrasound. Am J Respir Crit Care Med 2017; 196: e14.

17. Watanabe A, Taniguchi M, Kimura Y, Ito S, Hosokawa M, Sasaki S. Efficient, effective, safe procedure to identify nonrecurrent inferior laryngeal nerve during thyroid surgery. Head Neck 2016; 38: 573-7.

18. Qiao N, Wu LF, Gao W, et al. Anatomic characteristics, identification, and protection of the nonrecurrent laryngeal nerve during thyroidectomy. Otolaryngol Head Neck Surg 2017; 157: 210-6.

19. Gurleyik G, Torun M, Gurleyik E. Nonrecurrent laryngeal nerve: precise detection by electrophysiological nerve monitoring. Cureus 2018; 10: e2670.

20. Gong RX, Luo SH, Gong YP, et al. Prediction of nonrecurrent laryngeal nerve before thyroid surgery - experience with 1825 cases. J Surg Res 2014; 189: 75-80. 